\title{
CLINICAL PRESENTATION OF AUTOINFLAMMATORY DISEASES IN CHILDREN, ADOLESCENTS AND YOUNG ADULTS: A LATIN AMERICAN MULTICENTER STUDY
}

Daniela Gerent Petry Piotto"^, Pedro Henrique L. Carneiro', Katia Kozu², Nádia Emi Aikawa², María Martha Katsicas³, Sheila K. F. Oliveira ${ }^{4}$, Claudia S. Magalhães ${ }^{5}$, Ana Luiza G. Cunha ${ }^{6}$, Blanca Elena Bica ${ }^{4}$, Carlos Nobre Rabelo Junior ${ }^{7}$, Cristina Battagliotti $^{8}$, Erica Naomi N. Matos ${ }^{9}$, Flavia P. S. Teixeira Santos ${ }^{10}$, Flavio Roberto Sztajnbok ${ }^{4}$, Liliana Bezrodnik ${ }^{11}$, Marcia Bandeira ${ }^{12}$, Marta Cristine F. Rodrigues ${ }^{4}$, Pablo García Munittis ${ }^{13}$, Simone Appenzeller ${ }^{14}$, Teresa Cristina M. Robazzi ${ }^{15}$, Gleice Clemente ${ }^{1}$, Clovis Artur Silva², Maria Teresa Terreri'

1.Universidade Federal de São Paulo, São Paulo (SP), Brazil; 2.Universidade de São Paulo, São Paulo (SP), Brazil; 3.Hospital de Pediatria Garrahan, Buenos Aires, Argentina; 4.Universidade Federal do Rio de Janeiro, Rio de Janeiro (RJ), Brazil; 5.Universidade Estadual Paulista "Júlio de Mesquita Filho", Botucatu (SP), Brazil; 6.Hospital Infantil João Paulo II, Belo Horizonte (MG), Brazil; 7.Hospital Geral de Fortaleza, Fortaleza (CE), Brazil; 8. Hospital de Niños de Santa Fe "Dr. Orlando Alassia”, Santa Fe, Argentina; 9.Universidade Federal de Mato Grosso do Sul, Pioneiros (MS), Brazil; 10.Universidade Federal de Minas Gerais, Belo Horizonte (MG), Brazil; 11. Hospital de Niños Dr. Ricardo Gutiérrez, Buenos Aires, Argentina; 12. Hospital Pequeno Príncipe, Curitiba (PR), Brazil; 13.Hospital El Cruce - Nestor Kirchner, Buenos Aires, Argentina; 14.Universidade Estadual de Campinas, Campinas (SP), Brazil; 15.Universidade Federal da Bahia, Salvador (BA), Brazil.

*Corresponding author: danielapetry@gmail.com

\section{BACKGROUND}

Multicenter studies of autoinflammatory diseases (AID) are scarce in Latin American population. Given the importance of different disease prevalence and phenotypes worldwide, particularly between children and adolescents/young adults (AYA), we aimed to compare accrued signs and symptoms, laboratorial findings and treatments in AID patients grouped by age at diagnosis of disease and sex.

\section{METHODS}

This is an observational, descriptive study of a Latin American (LA) registry through a designed web system for data storage, collected between 2015 and 2018. The case-report forms were translated into Portuguese and Spanish languages, including demographic, clinical, laboratorial, genetic features and treatment.

\section{RESULTS}

We included 152 patients, being 51.3\% male and 75\% Caucasians. The median disease onset age was 2.1 years (0-15.6 years), median diagnosis age 6.9 years ( $0-21.9$ years); 111 (73\%) were children ( $0-9$ years) and 41 (27\%) AYA (10-21 years). Periodic fever, aphthous stomatitis, pharyngitis, and adenitis syndrome (PFAPA) was the most frequent diagnosis, followed by chronic non-bacterial osteomyelitis (CNO) and familial Mediterranean fever (FMF). Periodic fever, aphthous stomatitis, pharyngitis, and adenitis syndrome was seen more often in young children, and $\mathrm{CNO}$ in older patients, with female predominance. The FMF prevalence was higher in boys. Further analysis comparing children and AYA revealed that some clinical features, such as fever, cervical adenitis, aphthous stomatitis, pharyngitis and lymphadenopathy, were more frequent in children; and arthralgia, arthritis, bone pain, osteomyelitis, osteitis and aseptic arthritis were more frequent in AYA.

\section{CONCLUSION}

Differences related to accrued signs and symptoms, laboratorial findings and treatments in AID patients were observed between the LA population grouped by age at diagnosis of disease and sex. Children had predominantly PFAPA manifestations at presentation; whilst AYA had more musculoskeletal involvement.

\section{KEYWORDS}

Autoinflammatory disease, Children, Adolescents, Periodic fever, Familial Mediterranean fever. 